\title{
Direct Urca processes in superdense cores of neutron stars
}

\author{
L. B. Leinson \\ Institute of Terrestrial Magnetism, Ionosphere and Radio Wave \\ Propagation RAS, 142190 Troitsk, Moscow Region, Russia \\ E-mail: leinson@izmiran.rssi.ru
}

\begin{abstract}
We use the field theoretical model to perform relativistic calculations of neutrino energy losses caused by the direct Urca processes on nucleons in the degenerate baryon matter. By our analysis, in a free nucleon gas under beta equilibrium, the direct neutron decay is forbidden if the number density of neutrons exceeds the critical value $n_{n}^{c}=5.9 \times 10^{31} \mathrm{~cm}^{-3}$. In superdense nuclear matter, $n>n_{0}$, the weak decay of neutrons is possible only due to strong interactions, caused by an exchange of isovector mesons. Mean field of isovector mesons in the medium creates a large energy gap between spectrums of protons and neutrons, which is required by kinematics of beta decay. Our expression for the neutrino energy losses, obtained in the mean field approximation, incorporates the effects of nucleon recoil, parity violation, weak magnetism, and pseudoscalar interaction. For numerical testing of our formula, we use a self-consistent relativistic model of the multicomponent baryon matter. The relativistic emissivity of the direct Urca reactions is found substantially larger than predicted in the non-relativistic approach. We found that, due to weak magnetism effects, relativistic emissivities increase by approximately $40-50 \%$.
\end{abstract}

PACS number(s): 97.60.Jd , 21.65+f , 95.30.Cq

Keywords: Neutron star, Neutrino radiation

Typeset using REVTEX

*Talk given at the International Workshop "HADRON PHYSICS 2002", April 14 - 19, Bento Gonçalves, Rio Grande do Sul, Brazil. 
The simplest neutrino-emitting processes by which neutron stars can lose their thermal energy are beta decay of neutrons and electron capture on protons $n \rightarrow p+l+\bar{\nu}_{l}, \quad p+l \rightarrow$ $n+\nu_{l}$. These reactions, widely known as the direct Urca processes, are a central point of any modern scenarios of evolution of neutron stars because the neutrino energy losses caused by the direct Urca processes lead to a rapid cooling of the degenerate neutron star core. In the non-relativistic approximation, the energy losses caused by the direct Urca processes have been calculated more than ten years ago [1]

$$
Q_{n r}=\frac{457 \pi}{10080} G_{F}^{2} C^{2}\left(C_{V}^{2}+3 C_{A}^{2}\right) T^{6} M_{n}^{*} M_{p}^{*} \mu_{e} \Theta\left(p_{e}+p_{\mathrm{p}}-p_{\mathrm{n}}\right),
$$

Here $G_{F}$ is the Fermi weak coupling constant; $C=\cos \theta_{C}=0.973$ is the Cabibbo factor; $C_{V}=1$ and $C_{A} \simeq 1.26$ are the nucleon weak coupling constants. Here $M_{n}^{*}$ and $M_{p}^{*}$ are the effective nucleon masses, and $\mu_{e}$ is the electron chemical potential.

The energy exchange in the matter goes naturally on the temperature scale $\sim T$, which is small compared to typical kinetic energies of degenerate particles. Therefore the momenta of in-medium fermions are fixed at their values at Fermi surfaces, which we denote as $p_{\mathrm{n}}, p_{\mathrm{p}}$ for the nucleons and $p_{e}$ for electrons respectively. Then the "triangle" condition, $p_{\mathrm{p}}+p_{e}>p_{\mathrm{n}}$, required by the step-function, is necessary for conservation of the total momentum in the reaction and, together with the condition of charge neutrality of the medium, results in the threshold dependence on the proton concentration.

In spite of widely adopted importance of the direct Urca reactions, the corresponding neutrino energy losses are not well investigated yet. The simple formula (1) has been derived in the non-relativistic manner by neglecting all interactions between nucleons. Actually, however, the superthreshold proton fraction, necessary for the direct Urca processes to operate in the degenerate nuclear matter, appears at large densities, where the Fermi momenta of participating nucleons are comparable with their effective mass. Moreover, according to modern numerical simulations, the central density of the star can be up to eight times larger than the nuclear saturation density. This implies a substantially relativistic motion of nucleons in the superdense neutron star core. The appropriate equation of state for such a matter is actually derived in the relativistic approach, and the relevant neutrino energy losses must be consistent with the used relativistic equation of state.

We represent the totally relativistic consideration of the direct Urca processes on nucleons. Let us consider first the relativistic kinematics of the neutron beta decay $n \rightarrow p+l+\bar{\nu}_{l}$ in the degenerate matter under beta equilibrium. In what follows we consider massless neutrinos of energy and momentum $k_{1}=\left(\omega_{1}, \mathbf{k}_{1}\right)$ with $\omega_{1}=\left|\mathbf{k}_{1}\right|$. The energy-momentum of the final lepton $l=e^{-}, \mu^{-}$of mass $m_{l}$ is denoted as $k_{2}=\left(\omega_{2}, \mathbf{k}_{2}\right)$ with $\omega_{2}=\sqrt{\mathbf{k}_{2}^{2}+m_{l}^{2}}$. Thus, the energy and momentum conservation in the beta decay is given by the following equations

$$
\begin{array}{r}
E_{\mathrm{n}}(\mathbf{p})-E_{\mathrm{p}}\left(\mathbf{p}^{\prime}\right)-\omega_{1}-\omega_{2}=0 \\
\mathbf{p}-\mathbf{p}^{\prime}-\mathbf{k}_{1}-\mathbf{k}_{2}=0,
\end{array}
$$

where $E_{\mathrm{n}}(\mathbf{p})$ and $E_{\mathrm{p}}\left(\mathbf{p}^{\prime}\right)$ are the in-medium energies of the neutron and the proton respectively.

Since the antineutrino energy is $\omega_{1} \sim T$, and the antineutrino momentum $\left|\mathbf{k}_{1}\right| \sim T$ is much smaller than the momenta of other particles, we can neglect the neutrino contributions 
in Eqs. (2). Then the momentum conservation, $\mathbf{p}_{\mathrm{p}}+\mathbf{p}_{l}=\mathbf{p}_{\mathrm{n}}$, implies the well known "triangle" condition, $p_{\mathrm{p}}+p_{l}>p_{\mathrm{n}}$, necessary for the Urca processes to operate. However, the momentum conservation is necessary but insufficient condition for the direct beta decay to occur. The energy conservation requires a one more condition

$$
E_{\mathrm{n}}\left(p_{\mathrm{n}}\right)-E_{\mathrm{p}}\left(p_{\mathrm{p}}\right)=\sqrt{\left(\mathbf{p}_{\mathrm{n}}-\mathbf{p}_{\mathrm{p}}\right)^{2}+m_{l}^{2}}
$$

Squaring of both sides of this equation gives

$$
\left(E_{\mathrm{n}}\left(p_{\mathrm{n}}\right)-E_{\mathrm{p}}\left(p_{\mathrm{p}}\right)\right)^{2}-\left(\mathbf{p}_{\mathrm{n}}-\mathbf{p}_{\mathrm{p}}\right)^{2}=m_{l}^{2} .
$$

If we denote as

$$
K=\left(E_{\mathrm{n}}-E_{\mathrm{p}}, \mathbf{p}_{\mathrm{n}}-\mathbf{p}_{\mathrm{p}}\right),
$$

the energy-momentum transferred from the nucleon, this equation can be readily recognized as the following condition $K_{\mu}^{2}=m_{l}^{2}$. This condition naturally arises from the time-like momentum of the final lepton pair, $K=k_{1}+k_{2} \simeq k_{2}$, and can be satisfied only in the presence of the energy gap between spectrums of protons and neutrons. Really, consider the neutron decay in the degenerate matter. In this case the neutron from the neutron Fermi surface $E_{F n}$ undergoes transition into the proton at the proton Fermi energy $E_{F p}$.

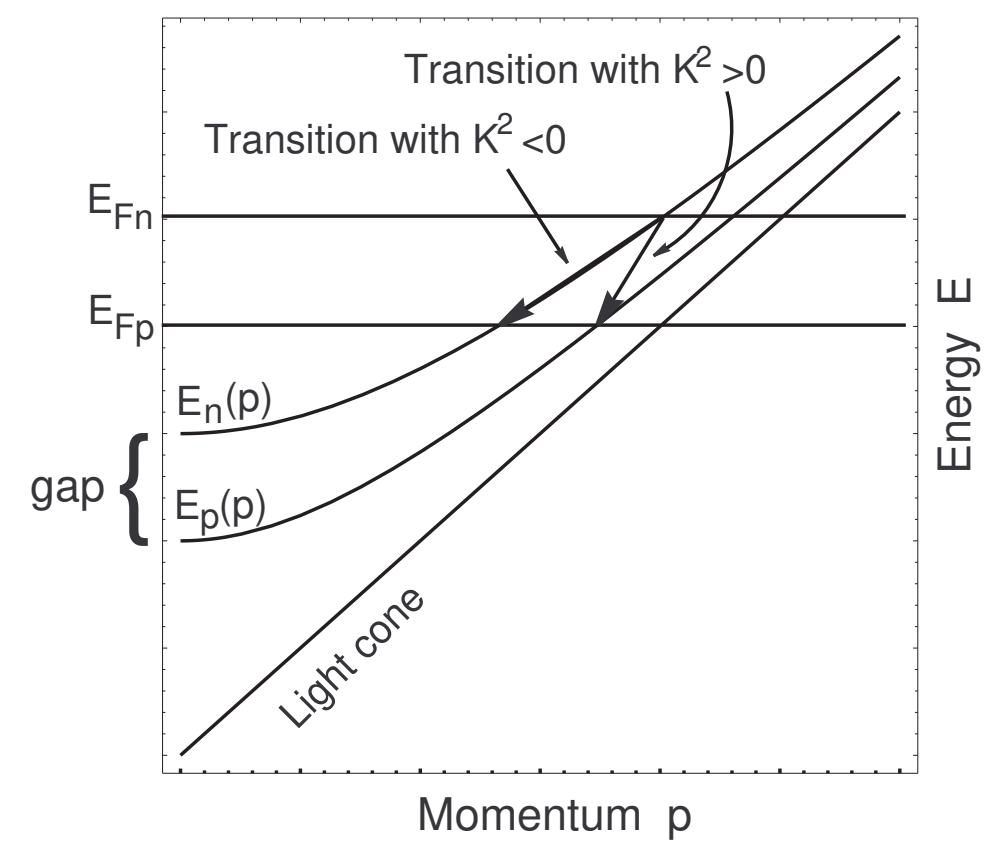

Fig. 1. The nucleon transition caused bu the beta decay. If the neutron and proton energy spectrums are identical (the upper curve), the momentum transfer due to such a transition is space-like. The energy gap between protons and neutrons allows the time-like momentum transfer to the leptons.

If the neutron and proton energy spectrums are identical (the upper curve, for example), the momentum transfer due to such a transition is space-like contrary to the time-like 
momentum of the final lepton pair. In this case the neutron decay is kinematically forbidden. In contrast, the energy gap between protons and neutrons allows the time-like momentum transfer to the leptons, and thus opens the direct neutron decay. It should be emphasized however that the stated conditions are consistent only in the presence of the energy gap between protons and neutrons. Therefore the possibility of the direct neutron decay depends substantially on the model of nuclear matter.

Consider, for example, a degenerate free gas consisting of neutrons, protons, and electrons under beta equilibrium. In this case the energy gap exists only due to the mass difference of neutron and proton. If we denote the masses as $M_{\mathrm{n}}$ and $M_{\mathrm{p}}$ respectively, then the corresponding Fermi energies are of the following relativistic form

$$
E_{\mathrm{n}}\left(p_{\mathrm{n}}\right)=\sqrt{M_{\mathrm{n}}^{2}+p_{\mathrm{n}}^{2}}, \quad E_{\mathrm{p}}\left(p_{\mathrm{p}}\right)=\sqrt{M_{\mathrm{p}}^{2}+p_{\mathrm{p}}^{2}} .
$$

Due to charge neutrality the number density of electrons, $n_{e} \propto p_{l}^{3}$, equals to the number density of protons, $n_{\mathrm{p}} \propto p_{\mathrm{p}}^{3}$. This implies equality of the proton and electron Fermi momenta, $p_{\mathrm{e}}=p_{\mathrm{p}}$, so that the "triangle" condition reads $2 p_{\mathrm{p}}=p_{\mathrm{n}}$. Neutrinos and antineutrinos freely escape from the neutron star. Then the chemical potential of neutrinos equals zero, $\mu_{\nu}=0$, and the condition of chemical equilibrium can be written as $\mu_{p}+\mu_{e}=\mu_{n}$. Chemical potentials of degenerate particles can be approximated by their individual Fermi energies, yielding

$$
\sqrt{m_{\mathrm{e}}^{2}+p_{\mathrm{p}}^{2}}+\sqrt{M_{\mathrm{p}}^{2}+p_{\mathrm{p}}^{2}}=\sqrt{M_{\mathrm{n}}^{2}+p_{\mathrm{n}}^{2}}
$$

Solution of this equation

$$
p_{\mathrm{p}}\left(p_{\mathrm{n}}\right)=\frac{1}{2 \sqrt{M_{\mathrm{n}}^{2}+p_{\mathrm{n}}^{2}}} \sqrt{\left(p_{\mathrm{n}}^{2}+M_{\mathrm{n}}^{2}-\left(M_{\mathrm{p}}+m_{\mathrm{e}}\right)^{2}\right)\left(p_{\mathrm{n}}^{2}+M_{\mathrm{n}}^{2}-\left(M_{\mathrm{p}}-m_{\mathrm{e}}\right)^{2}\right)}
$$

gives the proton Fermi momentum as a function of neutron Fermi momentum in the betaequilibrated gas of protons and neutrons. This function is shown by solid line.

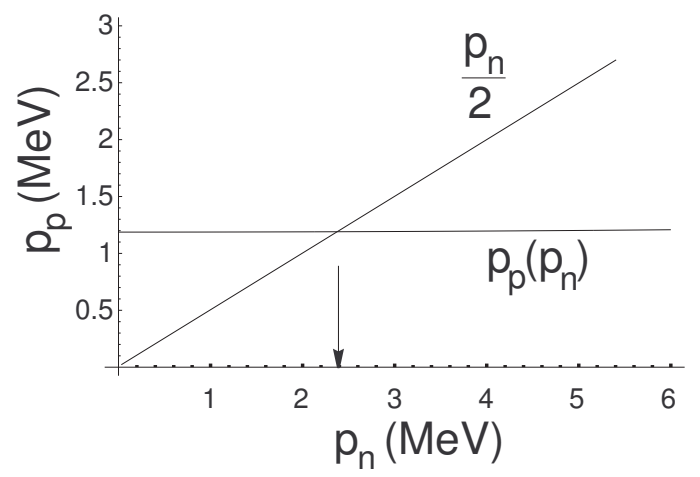

Fig. 2. Solid line is the proton Fermi momentum as a function of neutron Fermi momentum in the beta-equilibrated gas of protons and neutrons. Long-dashed line is $p_{\mathrm{n}} / 2$. The arrow shows the critical value of neutron Fermi momentum above which the direct neutron decay is forbidden. 
As required by the "triangle" condition, $2 p_{\mathrm{p}} \geq p_{\mathrm{n}}$, the direct neutron decay in such a medium is open when the proton Fermi momentum, $p_{\mathrm{p}}$, is larger than one half of the neutron Fermi momentum, $p_{\mathrm{n}} / 2$. This function is shown by long-dashed line. We can see, the direct neutron decay is allowed to the left of the crossing point and is forbidden at the right-hand side. The crossing point can be found as solution of the following equation

$$
p_{\mathrm{p}}\left(p_{\mathrm{n}}\right)=\frac{p_{\mathrm{n}}}{2}
$$

That is the critical value of the neutron Fermi momentum above which the direct neutron decay is forbidden

$$
p_{\mathrm{n}}^{c}=\frac{\sqrt{\left(\left(M_{p}+M_{n}\right)^{2}-m_{l}^{2}\right)\left(\left(M_{n}-M_{p}\right)^{2}-m_{l}^{2}\right)}}{\sqrt{2 M_{p}^{2}+2 m_{l}^{2}-M_{n}^{2}}}=2.3811 \mathrm{MeV} .
$$

Thus the direct neutron decay is forbidden if the number density of neutrons exceeds some critical value shown here

$$
n_{n}^{c}=\frac{\left(p_{\mathrm{n}}^{c}\right)^{3}}{3 \pi^{2}}=5.932 \times 10^{31} \mathrm{~cm}^{-3}
$$

This number density is much smaller than that typical for neutron star cores, therefore the direct neutron decay in the cooling neutron stars can occur only due to strong interactions.

Notice, some of models of strong interaction also forbid the direct neutron decay. Consider, for example, a simple model for the baryon matter, which contains fields for baryons and neutral scalar $(\sigma)$ and vector $\left(\omega_{\mu}\right)$ mesons. The isoscalar mesons equally interact with protons and neutrons, which therefore have identical energy spectrums. As discussed, in this case, the energy and the momentum in the direct neutron decay can not be conserved simultaneously.

To allow the direct neutron decay, the model of nuclear matter must be generalized to include some additional degrees of freedom and couplings, which are able to create a large energy gap between possible energies of protons and neutrons. For this purpose, besides isoscalar mesons $\sigma$ and $\omega$, the model should include also isovector mesons. The isovector meson couples differently to protons and neutrons, thus creating the energy gap necessary for the direct neutron decay.

In the following we consider a self-consistent relativistic model of nuclear matter in which baryons, $B=n, p, \Sigma^{-}, \Sigma^{0}, \Sigma^{+}, \Lambda$, interact via exchange of $\sigma, \omega$, and $\rho$ mesons [2]. The Lagrangian density,

$$
\begin{aligned}
\mathcal{L}= & \sum_{B} \bar{B}\left[\gamma_{\mu}\left(i \partial^{\mu}-g_{\omega B} \omega^{\mu}-\frac{1}{2} g_{\rho B} \mathbf{b}^{\mu} \cdot \tau\right)-\left(M_{B}-g_{\sigma B} \sigma\right)\right] B \\
& -\frac{1}{4} F_{\mu \nu} F^{\mu \nu}+\frac{1}{2} m_{\omega}^{2} \omega_{\mu} \omega^{\mu}-\frac{1}{4} \mathbf{B}_{\mu \nu} \mathbf{B}^{\mu \nu}+\frac{1}{2} m_{\rho}^{2} \mathbf{b}_{\mu} \mathbf{b}^{\mu} \\
& +\frac{1}{2}\left(\partial_{\mu} \sigma \partial^{\mu} \sigma-m_{\sigma}^{2} \sigma^{2}\right)-U(\sigma)+\bar{l}\left(i \gamma_{\mu} \partial^{\mu}-m_{l}\right) l,
\end{aligned}
$$

includes the interaction of baryon fields $B$ with a scalar field $\sigma$, a vector field $\omega_{\mu}$ and an isovector field $\mathbf{b}_{\mu}$ of $\rho$-meson. In the above, $B$ are the Dirac spinor fields for baryons, $\mathbf{b}_{\mu}$ 
is the isovector field of $\rho$-meson. We denote as $\tau$ the isospin operator, which acts on the baryons of the bare mass $M_{B}$. The leptons are represented only by electrons and muons, $l=e^{-}, \mu^{-}$, which are included in the model as noninteracting particles. The field strength tensors for the $\omega$ and $\rho$ mesons are $F_{\mu \nu}=\partial_{\mu} \omega_{\nu}-\partial_{\nu} \omega_{\mu}$ and $\mathbf{B}_{\mu \nu}=\partial_{\mu} \mathbf{b}_{\nu}-\partial_{\nu} \mathbf{b}_{\mu}$, respectively. The potential $U(\sigma)$ represents the self-interactions of the scalar field and is taken to be of the form

$$
U(\sigma)=\frac{1}{3} b M\left(g_{\sigma N} \sigma\right)^{3}+\frac{1}{4} c\left(g_{\sigma N} \sigma\right)^{4} .
$$

The parameters of the model are chosen as suggested in Ref. [3] to reproduce the nuclear matter equilibrium density, the binding energy per nucleon, the symmetry energy, the compression modulus, and the nucleon effective mass at saturation density $n_{0}=0.16 \mathrm{fm}^{-3}$.

In the mean field approximation, when the contribution of mesons reduce to classical condensate fields $\langle\sigma\rangle=\sigma_{0},\left\langle\omega^{\mu}\right\rangle=\omega_{0} \delta^{\mu 0},\left\langle\mathbf{b}^{\mu}\right\rangle \equiv\left(0,0, \rho_{0}\right) \delta^{\mu 0}$, only the baryon fields must be quantized. This procedure yields the following linear Dirac equation for the nucleon

$$
\left(i \partial_{\mu} \gamma^{\mu}-g_{\omega} \gamma^{0} \omega_{0}-\frac{1}{2} g_{\rho} \gamma^{0} \rho_{0} \tau_{3}-\left(M-g_{\sigma} \sigma_{0}\right)\right) \Psi(x)=0,
$$

The stationary and uniform condensate fields equally shift the effective masses

$$
M^{*}=M-g_{\sigma} \sigma_{0}
$$

but lead to different potential energies of protons and neutrons

$$
U_{\mathrm{n}}=g_{\omega} \omega_{0}-\frac{1}{2} g_{\rho} \rho_{0}, \quad U_{\mathrm{p}}=g_{\omega} \omega_{0}+\frac{1}{2} g_{\rho} \rho_{0},
$$

thus creating the energy gap $U_{\mathrm{n}}-U_{\mathrm{p}}=-g_{\rho} \rho_{0}$ between possible energies of protons and neutrons.

In the lowest order in the Fermi weak coupling constant $G_{F}$, the matrix element of the neutron beta decay is found to be

$$
\begin{aligned}
\mathcal{M}_{f i}= & -i \frac{G_{F} C}{\sqrt{2}} \bar{u}_{l}\left(k_{2}\right) \gamma_{\mu}\left(1+\gamma_{5}\right) \nu\left(-k_{1}\right) \times \\
& \times \bar{u}_{\mathrm{p}}\left(P^{\prime}\right)\left[C_{V} \gamma^{\mu}+\frac{1}{2 M} C_{M} \sigma^{\mu \nu} q_{\nu}+C_{A}\left(\gamma^{\mu} \gamma_{5}+F_{q} q^{\mu} \gamma_{5}\right)\right] u_{\mathrm{n}}(P),
\end{aligned}
$$

were $C=\cos \theta_{C}=0.973$ is the Cabibbo factor. This matrix element includes also the terms caused by weak magnetism and pseudoscalar interaction. In the mean field approximation, we assume $C_{V}=1, C_{M}=\lambda_{p}-\lambda_{n} \simeq 3.7, C_{A}=1.26$, and

$$
F_{q}=-\frac{2 M^{*}}{\left(m_{\pi}^{2}-q^{2}\right)} \text {. }
$$

In the above $\lambda_{p}$ and $\lambda_{n}$ are the anomalous magnetic moments of the proton and the neutron respectively.

Note that the matrix element (16) is of the same form as that for the neutron decay in a free space, but with the total momentum transfer replaced with the kinetic momentum transfer $q=(\varepsilon-\varepsilon, \mathbf{p}-\mathbf{p})$. 
We consider the total energy which is emitted into neutrino and antineutrino per unit volume and time. Within beta equilibrium, the inverse reaction $p+l \rightarrow n+\nu_{l}$ corresponding to a capture of the lepton $l$, gives the same emissivity as the beta decay, but in neutrinos. Thus, the total energy loss $Q$ for the Urca processes is twice more than that caused by the beta decay. Taking this into account by Fermi's "golden" rule we find:

$$
\begin{aligned}
Q= & \frac{457 \pi}{10080} G_{F}^{2} C^{2} T^{6} \Theta\left(p_{l}+p_{\mathrm{p}}-p_{\mathrm{n}}\right)\left\{\left(C_{A}^{2}-C_{V}^{2}\right) M^{* 2} \mu_{l}\right. \\
& +\frac{1}{2}\left(C_{V}^{2}+C_{A}^{2}\right)\left[4 \varepsilon_{\mathrm{n}} \varepsilon_{\mathrm{p}} \mu_{l}-\left(\varepsilon_{\mathrm{n}}-\varepsilon_{\mathrm{p}}\right)\left(\left(\varepsilon_{\mathrm{n}}+\varepsilon_{\mathrm{p}}\right)^{2}-p_{l}^{2}\right)\right] \\
& +C_{V} C_{M} \frac{M^{*}}{2 M}\left[2\left(\varepsilon_{\mathrm{n}}-\varepsilon_{\mathrm{p}}\right) p_{l}^{2}-\left(3\left(\varepsilon_{\mathrm{n}}-\varepsilon_{\mathrm{p}}\right)^{2}-p_{l}^{2}\right) \mu_{l}\right] \\
& +C_{A}\left(C_{V}+\frac{M^{*}}{M} C_{M}\right)\left(\varepsilon_{\mathrm{n}}+\varepsilon_{\mathrm{p}}\right)\left(p_{l}^{2}-\left(\varepsilon_{\mathrm{n}}-\varepsilon_{\mathrm{p}}\right)^{2}\right) \\
& +C_{M}^{2} \frac{1}{16 M^{2}}\left[8 M^{* 2}\left(\varepsilon_{\mathrm{n}}-\varepsilon_{\mathrm{p}}\right)\left(p_{l}^{2}-\left(\varepsilon_{\mathrm{n}}-\varepsilon_{\mathrm{p}}\right) \mu_{l}\right)\right. \\
& +\left(p_{l}^{2}-\left(\varepsilon_{\mathrm{n}}-\varepsilon_{\mathrm{p}}\right)^{2}\right)\left(2 \varepsilon_{\mathrm{n}}^{2}+2 \varepsilon_{\mathrm{p}}^{2}-p_{l}^{2}\right) \mu_{l} \\
& \left.-\left(p_{l}^{2}-\left(\varepsilon_{\mathrm{n}}-\varepsilon_{\mathrm{p}}\right)^{2}\right)\left(\varepsilon_{\mathrm{n}}+\varepsilon_{\mathrm{p}}\right)^{2}\left(2 \varepsilon_{\mathrm{n}}-2 \varepsilon_{\mathrm{p}}-\mu_{l}\right)\right] \\
& \left.-C_{A}^{2} M^{* 2} \Phi\left(1+m_{\pi}^{2} \Phi\right)\left[\mu_{l}\left(\left(\varepsilon_{\mathrm{n}}-\varepsilon_{\mathrm{p}}\right)^{2}+p_{l}^{2}\right)-2\left(\varepsilon_{\mathrm{n}}-\varepsilon_{\mathrm{p}}\right) p_{l}^{2}\right]\right\}
\end{aligned}
$$

with $\Theta(x)=1$ if $x \geq 0$ and zero otherwise. In the above, $\varepsilon_{\mathrm{n}}$ and $\varepsilon_{\mathrm{p}}$ are the kinetic Fermi energies of neutrons and protons respectively, and the last term, with

$$
\Phi=\frac{1}{m_{\pi}^{2}+p_{l}^{2}-\left(\varepsilon_{\mathrm{n}}-\varepsilon_{\mathrm{p}}\right)^{2}},
$$

represents the contribution of the pseudoscalar interaction.

Neutrino energy losses caused by the direct Urca on nucleons depend essentially on the composition of beta-stable nuclear matter. In order to examine numerically the relativistic effects in the direct Urca processes, we consider first a simplified model for degenerate nuclear matter of the standard composition consisting on neutrons, protons, electrons, and muons under beta-equilibrium. The left panel of Fig. 3 shows the composition of neutrino-free matter in beta equilibrium among nucleons, electrons and muons. The fractions of the constituents are shown versus the baryon number density, in units of saturation density. The right panel represents the emissivity of the Urca process, as given by our formula in comparison with the emissivity calculated without the effects of weak magnetism and pseudo scalar interaction. The short-dashed curve is the non-relativistic emissivity. All the emissivities are given in units $10^{27} T_{9}^{6} \mathrm{erg} \mathrm{cm}^{-3} \mathrm{~s}^{-1}$, where the temperature $T_{9}=T / 10^{9} \mathrm{~K}$. Thus the curves demonstrate how the emissivity varies along with the matter density.

We can observe that the relativistic effects dramatically modify the emissivity. The non-relativistic approximation approaches our result only at densities much smaller than

\footnotetext{
${ }^{1}$ Here we rectify an error made in the journal version of the paper. The correct expression can be obtained from that published in [4], [5] by simple replacement $C_{M} \rightarrow C_{M} / 2$. The author is grateful to M. Prakash and S. Ratković who have pointed out this error.
} 
the threshold density, indicated by the vertical line. Due to the decrease of the nucleon effective mass, the non-relativistic formula predicts a decreasing of the emissivity as density increases above the threshold. In contrast, our formula shows a substantial increasing of the neutrino energy losses as we go to larger densities. The long-dashed curve demonstrates the energy losses obtained from the relativistic formula by formal setting $C_{M}$ equal zero and $\Phi$ equal zero (See also Ref. [6]). This eliminates the weak magnetism and pseudo scalar contributions. A comparison of the long-dashed and solid curve demonstrates that due to weak magnetism effects, relativistic emissivities increase by approximately $40-50 \%$.
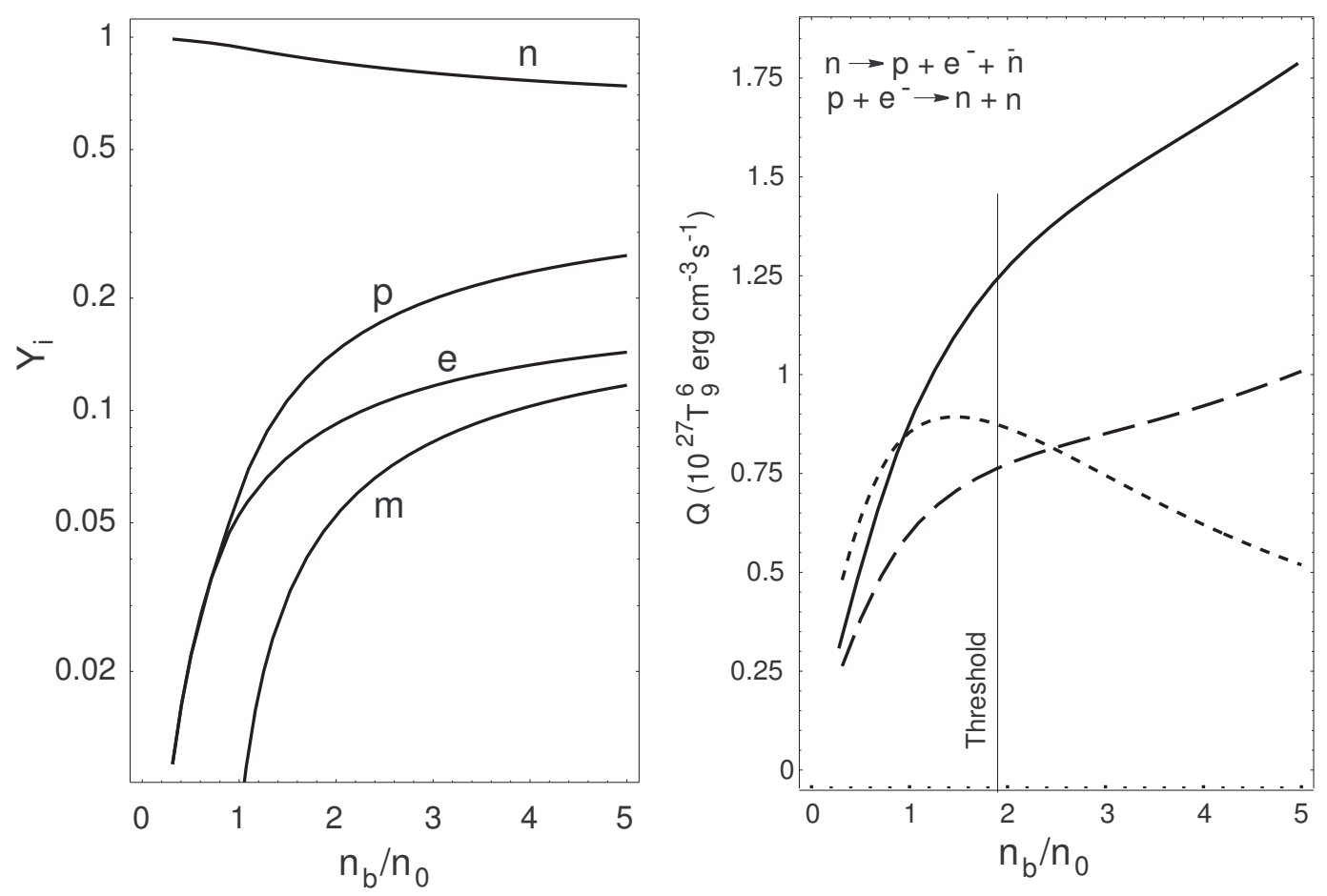

Fig. 3. The left panel shows the composition of neutrino-free matter in beta equilibrium among nucleons, electrons and muons. The fractions are shown versus the baryon number density, in units of saturation density. Right panel represents the emissivity of the Urca process, as given by our formula (solid curve) in comparison with the emissivity calculated without the effects of weak magnetism and pseudo scalar interaction (long-dased curve). The short-dashed curve is the non-relativistic emissivity. All the emissivities are given in units $10^{27} T_{9}^{6} \mathrm{erg} \mathrm{cm}^{-3} \mathrm{~s}^{-1}$, where the temperature $T_{9}=T / 10^{9} \mathrm{~K}$.

To examine dependence of energy losses on the matter composition we consider the model, which includes nucleon and hyperon degrees of freedom. The composition of neutrinofree matter in beta equilibrium among nucleons, hyperons, electrons and muons is shown in this figure versus the baryon number density. The right panel represents the emissivity of the direct Urca processes, as given by our formula in comparison with the emissivity calculated without the effects of weak magnetism and pseudo scalar. The short deshed curve is again the non-relativistic emissivity. We see that the neutrino energy losses caused by the direct 
Urca processes on nucleons depend essentially on the composition of beta-stable nuclear matter. Appearance of hyperons in the system suppresses the nucleon fractions and lepton abundance. Therefore at densities, where the number of hyperons is comparable with the number of protons, the relativistic emissivity reaches the maximum and then has a tendency to decrease. The relativistic emissivity, however, is found to be substantially larger than that predicted in the non-relativistic approach. The weak magnetism effects again approximately doubles the relativistic emissivity.
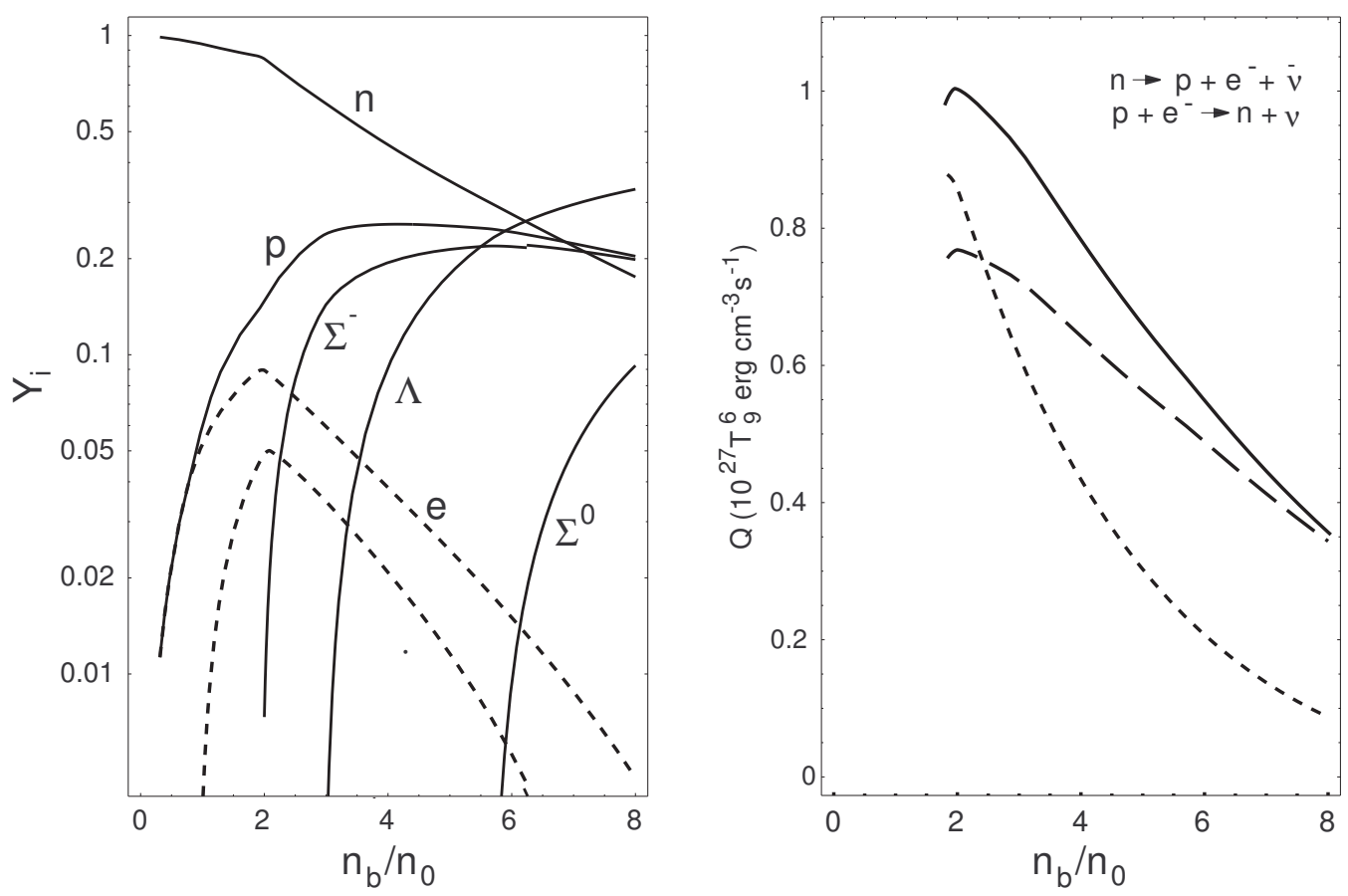

Fig. 4. The same as Fig. 3 but for the matter composition including hyperons.

Let's summarize our results. We have shown that the direct Urca processes in a superdense matter of neutron star cores are kinematically allowed only due to isovector mesons, which differently interact with protons and neutrons. By creating the energy gap between proton and neutron spectrums the isovector mesons support a time-like total momentum transfer from the nucleon, as required by kinematics of the reaction. In the mean field approximation, we derived the matrix element of the nucleon transition current, which is found to be a function of the space-like kinetic momentum transfer. In the mean field approximation, we have calculated the neutrino energy losses caused by the direct Urca processes on nucleons. Our Eq. (18) for neutrino energy losses exactly incorporates the effects of nucleon recoil, parity violation, weak magnetism, and pseudoscalar interaction. To quantify the relativistic effects we consider a self-consistent relativistic model, widely used in the theory of relativistic nuclear matter. The relativistic energy losses are up to four times larger than those given by the non-relativistic approach. In our analysis, we pay special attention to the effects of weak magnetism and pseudoscalar interaction in the neutrino energy losses. We found that due to weak magnetism effects, relativistic emissivities increase by approximately 40- 
$50 \%$. The efficiency of the direct Urca processes involving different kinds of baryons depends essentially on the composition of the beta-stable nuclear matter. The energy losses in the standard nuclear matter increase along with the density. In contrast, the total energy losses in the multicomponent medium have a tendency to decrease. 


\section{REFERENCES}

[1] J. M. Lattimer, C.J. Pethick, M. Prakash, and P. Haensel, Phys. Rev. Lett., 66 (1991) 2701.

[2] B. D. Serot and J. D. Walecka, Adv. Nucl. Phys. 19, eds. J.W. Negele and E. Vogt, (Plenum, New York, 1986); B.D. Serot, Rep. Prog. Phys. 55 (1992) 1855.

[3] N. K.Glendenning and S. A. Moszkowski, Phys. Rev. Lett. 67 (1991) 2414.

[4] L. B. Leinson, Phys. Lett. B 532 (2002) 267;

[5] L. B. Leinson, Nucl, Phys. A 707 (2002) 543.

[6] L. B. Leinson and A. Perez, Phys. Lett. B 518 (2001) 15; Erratum: Phys. Lett. B, 522 (2001) 358. 